\title{
Phenotypic variability in a family with Townes-Brocks syndrome
}

\author{
Yosuke Sudo $^{1}$, Chikahiko Numakura ${ }^{1}$, Akiko Abe ${ }^{1}$, Satoru Aiba ${ }^{2}$, Akira Matsunaga ${ }^{1}$ and Kiyoshi Hayasaka ${ }^{1}$ \\ Townes-Brocks syndrome (TBS) is an autosomal dominant disorder characterized by external ear anomalies with sensorineural \\ hearing loss, limb anomalies, renal and anorectal malformations. TBS is caused by mutations in SALL1, a gene mapped to \\ chromosome 16q12.1. We report three generations of a family with SALL1 c.1326delC (p.Ser442fs) mutation, showing \\ increased clinical severity over generations. The members of the first generation demonstrated polydactyly and deafness. \\ In the second generation, the mother and uncle of the proband additionally had renal and/or anal anomalies. The proband in the \\ third generation showed the most severe symptoms including congenital heart disease. Increase in clinical severity in successive \\ generations in TBS cannot be explained genetically. There is wide clinical variation in TBS; however, most affected parents are \\ usually mildly affected and may have similarly or more severely affected children. Social and/or physical bias at reproduction \\ may contribute to an apparent increase in clinical severity over generations in TBS.
}

Journal of Human Genetics (2010) 55, 550-551; doi:10.1038/jhg.2010.64; published online 3 June 2010

Keywords: SALL1; Townes-Brocks syndrome; phenotypic variability

\section{INTRODUCTION}

Townes-Brocks syndrome (TBS) is an autosomal dominant disorder characterized by first and second arch defects including auricular, renal, finger and anal anomalies. ${ }^{1}$ TBS is caused by mutations in SALL1, a gene related to the developmental regulator gene sal of Drosophila melanogaster. ${ }^{2}$ Most mutations are located between the glutamine-rich domain and the first double zinc finger domain within exon 2. ${ }^{3}$ Occasional abnormalities include foot anomalies, deafness, mental retardation, microcephaly, microtia, cardiac defect and hypothyroidism. ${ }^{1,4,5}$ Wide phenotypic variations are known even within a family with the same SALL1 mutations. ${ }^{6}$

Here, we report three generations of a family with TBS showing significant phenotypic variations.

\section{CLINICAL REPORT}

\section{Family report}

The proband (III-1) was a four-year-old Japanese girl. She was born at 41 weeks gestation after an uneventful pregnancy to a 29-year-old gravida 0 , para 0 mother. Her birth weight was $3030 \mathrm{~g}$ ( -0.2 s.d.), height was $49.7 \mathrm{~cm}$ (+0.4 s.d.) and head circumference was $32.5 \mathrm{~cm}$. She had bilateral preauricular tags, bilateral preaxial polydactyly, syndactyly of the right toe, second and fourth overlapping third of the toe on the left and anteriorly placed and stenosed anus. She also had mild bilateral sensorineural hearing loss. Ultrasonographic studies showed ventricular septal defect and small right kidney. The patient was diagnosed with TBS based on her clinical findings and her dominant familial history.
Her mother (II-1), maternal uncle (II-2), grandfather (I-1) and great aunt (I-3) were also affected (Figure 1). Her mother had right preaxial polydactyly, anterior placement of the anus, small right kidney and mild sensorineural hearing loss. The uncle had right preaxial polydactyly, imperforate anus and mild hearing loss. The grandfather and great aunt had only unilateral preaxial

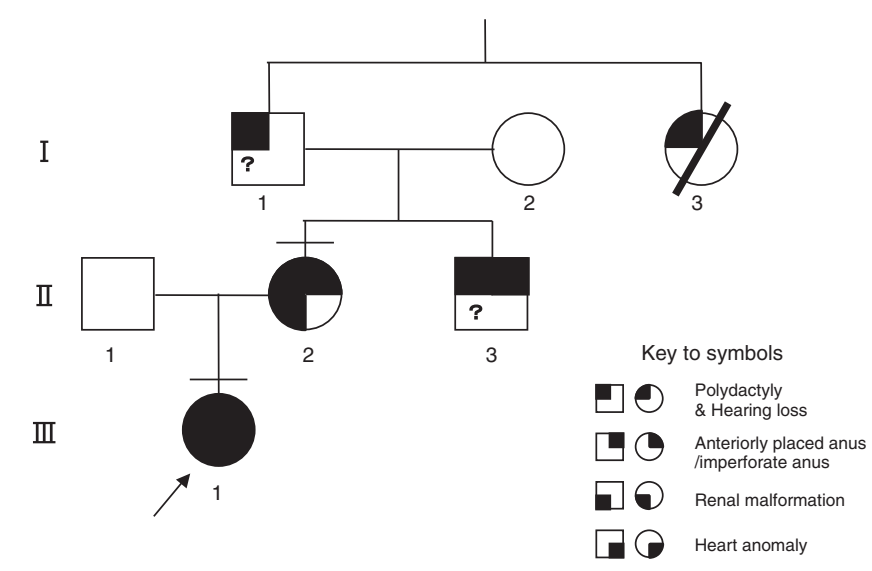

Figure $1 \mathrm{~A}$ family pedigree. Arrow indicates the proband. Clinical phenotypes are represented by symbols. Genetic analysis was performed in the proband (III-1) and her mother (II-2). Information for renal involvement was not available in the grandfather (I-1) and uncle (II-3).

${ }^{1}$ Department of Pediatrics, Yamagata University School of Medicine, Yamagata, Japan and ${ }^{2}$ Department of Pediatrics, Yamagata Prefectural Central Hospital, Yamagata, Japan Correspondence: Dr K Hayasaka, Department of Pediatrics, Yamagata University School of Medicine, 2-2-2 lida-nishi, Yamagata 990-9585, Japan. E-mail: hayasaka@med.id.yamagata-u.ac.jp

Received 8 December 2009; revised and accepted 12 May 2010; published online 3 June 2010 
polydactyly and deafness. Great aunt had renal failure due to chronic glomerulonephritis, was treated hemodialysis from age 37 and died of pancreas cancer at age 57 .

\section{MOLECULAR STUDIES}

All coding regions of exons 2 and 3 were amplified by polymerase chain reaction using primers designed based on the genome data and were directly sequenced. The sequence demonstrated that the proband (III-1) and mother (II-2) were heterozygotes for novel c.1326delC (p.Ser442fs) mutation.

\section{DISCUSSION}

We report a family with TBS demonstrating wide variations in phenotype. Wide phenotypic variations even within a family are clinical features of TBS. Kosaki et al. (2007) reported two sisters carrying a heterozygous $1256 \mathrm{~T}>\mathrm{A}$ (L419X) mutation of SALL1, one with a TWS phenotype and the other showing features of Goldenhar syndrome-like phenotype (epibulbar dermoid, bilateral microtia with atretic ear canals). ${ }^{6}$ The clinical presentation of TBS can overlap with Goldenhar syndrome, Okihiro syndrome, branchiootorenal syndrome and VACTERL association. The information of other family members and SALL1 mutation analysis helped us to confirm the diagnosis.

To date, $>50$ variants of $S A L L 1$ mutations have been reported. ${ }^{2,3,6-9}$ Most mutations are located between the glutamine-rich domain and the first double zinc finger domain within exon 2, a hot spot, as reported earlier. ${ }^{2,3}$ The proband and mother both demonstrated a novel deletion mutation, c.1326delC, which is also positioned within the hot spot in SALL1. Most SALL1 mutations result in premature termination codons that had been predicted to trigger nonsensemediated decay of mutant mRNA and cause haploinsufficiency. However, a mouse carrying a Sall1-null allele does not mimic the human TBS and a mouse carrying a Sall1 mutant allele that produces a truncated protein and presents clinical features of TBS. ${ }^{10}$ Kiefer $e t$ al. (2003) demonstrated that truncated Sall1 mediates interaction with all Sall family members and could interfere with the normal function of all Sall proteins. ${ }^{10}$ Furniss et al. (2007) reported a heterozygous 995delC mutation in exon 2 of SALL1 escaped nonsense-mediated decay and produced a truncated protein acting in a dominant-negative manner. ${ }^{11}$ Nonsense-mediated decay efficiency varies between individuals and is tissue specific, which may contribute to phenotypic variation in the patients carrying the same mutations.

The severity of phenotypes or numbers of involved organs in our family increased with each succeeding; that is, anticipation-like phenomenon. As shown in Figure 1, the grandfather (I-1) and great aunt (I-3) only demonstrated unilateral preaxial polydactyly and deafness. In addition to polydactyly and hearing disturbance, her mother (II-2) demonstrated anal and renal anomalies, and her uncle (II-3) had anal anomaly. Information for renal involvement was not available in the grandfather (I-1) and uncle (II-3). In this family, the proband (III-1) showed the most severe symptoms including congenital heart disease. An increase in the clinical severity has been observed in a number of families with TBS, frequently in patients that inherited the disease from their mothers. ${ }^{3,6,8,9,12,13}$ Anticipation is common in trinucleotide repeat disorders such as Huntington's disease and myotonic dystrophy where a dynamic mutation in DNA occurs. However, increased severity in TBS cannot be explained genetically, as triplet repeat disease. If SALL1 is expressed in the gametocye especially in the oocyte and mutant SALL1 affects the condition of the fertilized oocyte, anticipation-like phenomenon may occur. However, SALL1 is not expressed in the oocytes at fertilization. We should consider the social and/or physical bias at reproduction as another possibility, because most parents are usually only mildly affected as described in many reports and have similarly or more severely affected children. Wide clinical variation is a character of TBS caused by SALL1 mutations. Social and/or physical bias may contribute to an apparent increase in clinical severity over generations in TBS.

In genetic counseling, it is necessary to explain that patients with TBS present wide phenotypic variations even in the same family.

1 Powell, C. M. \& Michaelis, R. C. Townes-Brocks syndrome. J. Med. Genet. 36, 89-93 (1999).

2 Kohlhase, J., Wischermann, A., Reichenbach, H., Froster, U. \& Engel, W. Mutations in the SALL1 putative transcription factor gene cause Townes-Brocks syndrome. Nat. Genet. 18, 81-83 (1998).

3 Botzenhart, E. M., Bartalini, G., Blair, E., Brady, A. F., Elmslie, F., Chong, K. L. et al. Townes-Brocks syndrome: twenty novel SALL1 mutations in sporadic and familial cases and refinement of the SALL1 hot spot region. Hum. Mutat. 28, 204-205 (2007).

4 Ishikiriyama, S., Kudoh, F., Shimojo, N., Iwai, J. \& Inoue, T. Townes-Brocks syndrome associated with mental retardation. Am. J. Med. Genet. 61, 191-192 (1996).

5 Goswami, V. \& Dubev, N. Townes-Brocks syndrome with hypothyroidism. Indian Pediatr. 44, 140-142 (2007).

6 Kosaki, R., Fujimaru, R., Samejima, H., Yamada, H., Izumi, K., lijima, K. et al. Wide phenotypic variations within a family with SALL1 mutations: isolated external ear abnormalities to Goldenhar syndrome. Am. J. Med. Genet. 143, 1087-1090 (2007).

7 Liang, Y., Shen, D. \& Cai, W. Two coding single nucleotide polymorphisms in the SALL1 gene in Townes-Brocks syndrome: a case report and review of the literature. J. Pediatr. Surg. 43, 391-393 (2008).

8 Botzenhart, E. M., Green, A., Ilyina, H., König, R., Lowry, R. B., Lo, I. F. et al. SALL1 mutation analysis in Townes-Brocks syndrome: twelve novel mutations and expansion of the phenotype. Hum. Mutat. 26, 282-290 (2005).

9 Blanck, C., Kohlhase, J., Engels, S., Burfeind, P., Engel, W., Bottani, A. et al. Three novel SALL1mutations extend the mutational spectrum in Townes-Brocks syndrome. J. Med. Genet. 37, 303-307 (2000).

10 Kiefer, S. M., Ohlemiller, K. K., Yang, J., McDill, B. W., Kohlhase, J. \& Rauchman, M. Expression of a truncated Sall1 transcriptional repressor is responsible for TownesBrocks syndrome birth defects. Hum. Mol. Genet. 12, 2221-2227 (2003).

11 Furniss, D., Critchley, P., Giele, H. \& Wilkie, A. O. Nonsense-mediated decay and the molecular pathogenesis of mutations in SALL1 and GLI3. Am. J. Med. Genet. A. 143A, 3150-3160 (2007).

12 Surka, W. S., Kohlhase, J., Neunert, C. E., Schneider, D. S. \& Proud, V. K. Unique family with Townes-Brocks syndrome, SALL1 mutation, and cardiac defects. Am. J. Med. Genet. 102, 250-257 (2001).

13 Kohlhase, J., Liebers, M., Backe, J., Baumann-Muller, A., Bembea, M., Destree, A. et al. High incidence of the R276X SALL1 mutation in sporadic but not familial Townes-Brocks syndrome and report of the first familial case. J. Med. Genet. 40, e127 (2003). 